\section{Covering the Universe}

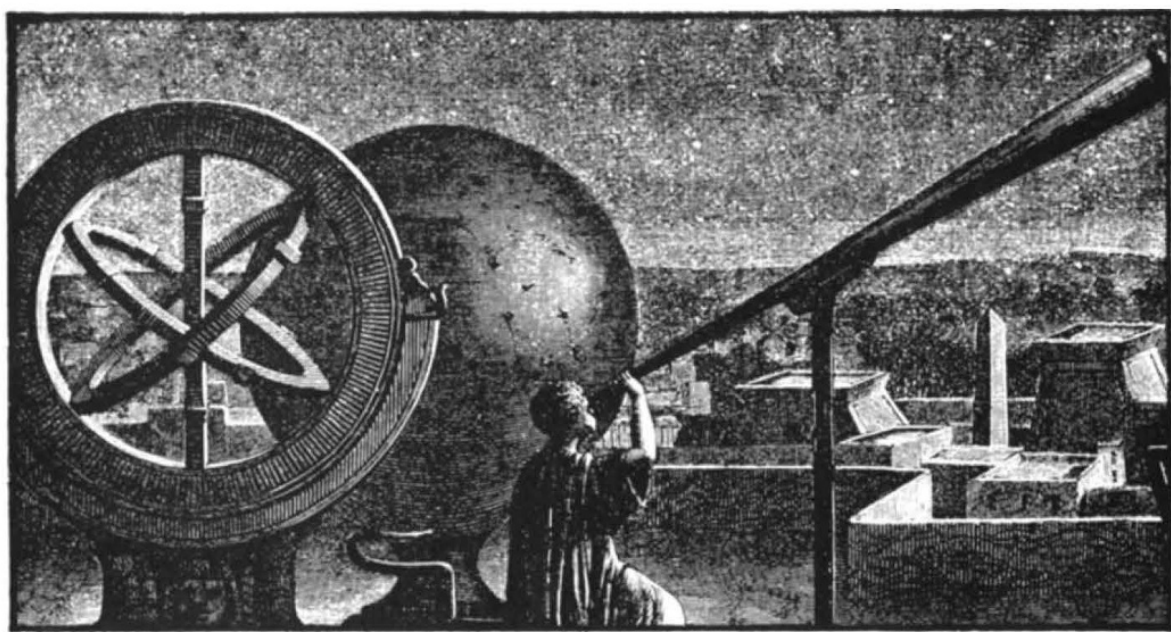

Astrophysics of Gaseous Nebulae. By Donald E. Osterbrock. Pp. xiv +251 . (Freeman : San Francisco and Reading, January 1975.) $£ 8.90$.

IN the Milky Way and in other galaxies the very brightest stars are generally surrounded by masses of bright interstellar gas. These are the H II regions, so called because the hydrogen there is in the form of $\mathrm{H}^{+}$. Other atomic species, notably helium, are also largely ionised as well.

All of these ions are continually recombining with the electrons present. If recombination takes place to an excited state then the resultant downward cascade of the recombined atom or ion produces a rich spectrum of emission lines. The optical observation of HII regions relies on these lines; the radio observer can also detect spectral lines arising from transitions between very high levels of the atom concerned. H II regions show up in the radio continuum as well. The emission process here is free-free emission by an electron in the field of an ion.

In principle, the observations contain an enormous wealth of information about the density, temperature, and composition of the gas in $\mathrm{H}$ II regions, and its distribution in space. But to tap this wealth requires a considerable amount of physical analysis. Much of the required work has been done by Don Osterbrock and his colleagues; in this book he presents an account of the subject which is so good that it should be unnecessary from now on to consult any earlier work.

The processes by which an equilibrium is attained in a diffuse mass of gas sitting around a bright star which is rich in ultraviolet radiation include radiative transfer and electron collision processes; since many different types of atom are involved the situation becomes quite complicated, but the author never lets it get out of hand. For good measure he also deals with planetary nebulae, where many of the same phenomena occur on a smaller scale.

But there have been many developments in our understanding of $\mathrm{H} \mathrm{II}$ regions since the text was completed (about three years ago) and in particular much attention is now being focused on compact $\mathrm{H} I \mathrm{II}$ regions. These are objects whose linear dimensions are smaller. They are often heavily obscured by dust, and are therefore observed mainly at radio and infrared wavelengths. There is a definite association between dense molecular clouds and these compact objects. Very probably they occur in regions where star formation is still active, and the stars involved in them must be among the youngest objects in the sky. Some of these developments are hinted at in the book, but I hope that Professor Osterbrock will be persuaded to write with these more recent discoveries.

F. D. Kahn Perspectives. By Cyril Ponnamperuma and A. G. W. Cameron. Pp. 226. (Houghton Mifflin: Boston, 1974.) $£ 3.50$.

ONLY two serious possibilities exist for acquiring information about extraterrestrial life. The first is by direct rocket flight within the Solar System. It is possible, though by no means sure, that one of our sister planets may possess a primitive biosphere. The second is by interstellar communication with remote technical communities elsewhere in the Galaxy. With the declining interest in the spaceflight programme, the exciting if somewhat exotic, possibility of radio communicaa second, more detailed, volume to deal

Interstellar Communication: Scientific tion with extraterrestrial civilisations is achieving mounting respectability and being given serious consideration by astronomers, biochemists, physicists and even social scientists.

In the summer of 1970 a conference took place at NASA's Ames Research Center to bring together acknowledged experts on this nascent subject. The presentations at that conference have now been made available in this book. Enthusiasts will recognise the names of the contributors-Sagan, Drake, Bracewell, Arbib, McCarthy, Aranoff, Oliver and Morrison, as well as those of the editors, Ponnamperuma and Cameron. The book is a well-balanced and eminently readable account of all the varying aspects of this intriguing and daring topic. Although this work constitutes a serious and in-depth study, including technical material of interest to experimentalists, the informal style and interdisciplinary profile of the subject matter will command a wide readership from non-specialists of many disciplines. (It also contains a 39 page bibliography.)

The feasibility of radio communication with extraterrestrial civilisations depends heavily on the proportion of stars in the Galaxy which are likely to support life-bearing planets with technical communities capable of transmitting, receiving and interpreting radio signals. Surprisingly, the most uncertain parameter which determines this proportion turns out to be a social onethe mean lifetime of technical communities. The contributors apparently share a strong opinion that the physical and biological conditions necessary for the formation of life are extremely common throughout the Universe and that given the appropriate type of star, intelligent life of some sort is almost inevitable. Carl Sagan comes up with a formula which predicts that the number of technical communities in the Galaxy is $10 \%$ of their mean lifetime in years. This implies the curious result that either the Galaxy is teeming with intelligent life, or, if our civilisation is typical, we are already doomed.

With the optimistic assumption that the technical phase lasts billions of years (there is plenty of time available since the Galaxy was formed) then there may be a few hundred million communicative planets around us.

The formidable problem which then emerges is where to look. Even the optimistic estimates indicate the necessity for a search pattern covering thousands of stars out to a distance of perhaps 1,000 light years-a daunting prospect. Nevertheless, in the early 1960s Frank Drake monitored two nearby stars at $21 \mathrm{~cm}$ wavelength in the famous Project Ozma, in the hope of detecting intelligently directed signals. A vastly more ambitious programme is 